\title{
A Dynamic Factor Model for Italian and German Recessions
}

\author{
Giovanni De Luca \\ University of Naples Parthenope \\ Alfonso Carfora \\ Italian Revenue Agency
}

\begin{abstract}
In this paper we analyze a model for the prediction of Italian and German recessions making use of two macro factors that summarize a high number of variables. The two factors consist of a financial factor and real activity factor. Their dynamics is described by random walks. The results are compared with some of the Kauppi and Saikkonen (2008) models. While the in-sample performance of the Dynamic Autoregressive model by Kauppi and Saikkonen is unrivalled, the 6 and 12-months horizons out-of-sample analysis favors the models including some of the factors.
\end{abstract}

Keywords: Business Cycles; Forecasting; Dynamic Factors; Probit Model JEL Codes: E32; C22; C25

\section{Introduction}

The modelling and prediction of economic recessions is a topic largely debated among policy makers and researchers. In the recent years a huge amount of attention has been devoted to the last economic recession. Starting from United States, the recession has transmitted to the European countries because of the strict links between the markets. So, both U.S. and European researchers have contributed to the increasing number of studies focusing on the analysis of the causes and on the prediction of recessions.

From a statistical point of view, the recession is treated as a binary random variable, assuming value 0 (absence of recession) and 1 (presence of recession). So, all statistical models thought for such random variables can be potentially adopted for modelling recessions.

In literature, there are a number of relevant contributions. Estrella and Mishkin (1998) have considered some financial variables (such as interest rates, stock prices and so on) in a probit framework to predict recessions. Chauvet and Potter (2005) have generalized the probit model allowing for autocorrelation of the errors and possible break points. Kauppi and Saikkonen (2008) have proposed to extend the static formulation including both lagged values of the binary response and lagged values of the probit probability. Their dynamic models certainly provide accurate forecasts of U.S. recessions. Moreover, Startz (2008) has introduced the autopersistence functions driving towards models inspired by the ARMA philosophy, and finally Nyberg (2010) has applied dynamic probit models to U.S. and German recessions.

A recent stream of research suggests that the recession is a complex phenomenon and a large set of explanatory variables has to be considered to improve recession probabilities. This approach has been followed by Chen et al. (2011) applying a probit model with common factors extracted from a large set of explanatory variables and Fossati (2015) with the proposal of determining three macroeconomic factors related to the financial variables, the stock market indicators and the real activity. Finally, Fornaro (2016) has adopted a Bayesian methodology associated to a shrinkage techniques.

In this paper, we purse the idea of Fossati (2015) of building some macroeconomic factors as potential explanatory variables for predicting recessions. However, given that the observed variables used in the construction of the factors are mainly non stationary, and assumed a linear relationship between observables and factors, the dynamics of each factor is described as a random walk. The main aim of the analysis is the forecasting of the recession, especially in the yearly horizon. For this reason, we will choose 12-months lagged factors to explain present recession. 
The paper is organized as follows. In Section 2 the Dynamic Factor model is described and the construction of the two macroeconomic factors is detailed. Section 3 presents the Kauppi and Saikkonen (2008) model. The estimation of the models and the out-of-sample analysis can be found is in Section 4. Section 5 concludes.

\section{The DynamicFactor model}

In this work we use the business cycle dates diffused by ECRI to create the binary monthly time series of the recessions of Italy and Germany from 2002 to 2014. The series are created coding each month with 1 for recession, if it is identified as being in a recession by ECRI, with 0 otherwise.

As a result, the modelling of economic recessions claims a framework with a binary dependent variable. The most popular model which describes such a variable is the probit model which represent a benchmark in this context. The ability of the researcher is that of capturing the relevant variables entering the model able to explain the binary variable.

When the economic recession is the binary variable to model, a long list of possible financial and economic explanatory variables has to be considered. The selection of a few number of variables is then a difficult task. Alternatively, a reduction-of-dimensionality method can be considered with the benefit of including all the variables each with its own weight.

In this paper we pursue the second approach, because it is our opinion that the complexity of the phenomenon of recession could be disentangled exploiting a wide range of variables. To this aim we have created some latent factors using a Dynamic Factor model.

Let $X_{t}$ be a $n \times 1$ vector denoting $n$ observed variables at time $t$ and let $F_{t}$ be a univariate factor. Then the estimated Dynamic Factor model implies a state-space representation given by

$$
\begin{aligned}
& X_{t}=Z F_{t}+v_{t} \\
& F_{t}=T F_{t-1}+w_{t}
\end{aligned}
$$

where $v_{t}$ is a multivariate Gaussian with zero vector mean and $n \times n$ covariance matrix given by $Q$, while $w_{t}$ is a univariate Gaussian with zero mean and variance equal to $\sigma_{w}^{2}$. Moreover, $v_{t}$ and $w_{t}$ are uncorrelated. The $n \times 1$ vector Zrepresents the loadings of the factor. The parameter $T$ is constrained to be one, that is the factor is assumed to be a random walk. Its nonstationary nature is demanded by the non-stationarity of the observables which are summarized in the factor.

The Dynamic Factor model is applied for estimating two factors.

\subsection{The financialfactor}

The first factor is built using a high number of variables (16) concerning interest rates, exchange rates and stock market movements ${ }^{1}$. The most of them are common to Italy and Germany. The variables are:

1. European Central Bank interest rate (EURIBOR), common to Italy andGermany (source: Eurostat);

2. 12-months interest rate $(\mathrm{B} 12 \mathrm{~m})$ for the Italian BOT and the German Bond(source: Italian Ministry of Finance and Datastream, Thomson Reuters);

3. 10-years interest rate (B10y) for the Italian BTP and the German Bund (source: Eurostat);

4. Moody's AAA Corporate bond yield from Federal Reserve Economic Data(AAA), common to Italy and Germany. It is an investment bond that acts as an index of the performance of all bonds given an AAA rating by Moody's Investors Service (source: Federal Reserve Economic Data FRED St Louis);

5. Moody's BAA Corporate bond yield from Federal Reserve Economic Data(BAA), common to Italy and Germany (source: Federal Reserve Economic Data - FRED St Louis);

6. spread between 12-months BOT/Bond and Euribor (SprB12mE);

7. spread between 10-years BTP/Bund and Euribor (SprB10yE);

8. spread between Moody's AAA Corporate bond yield and Euribor (SprAAAE),common to Italy and Germany;

9. spread between Moody's BAA Corporate bond yield and Euribor (SprBAAE), common to Italy and Germany; 10.Real effective exchange rate producer prices deflated (ER), common toItaly and Germany (source: Eurostat); 11.Exchange rate between Euro and US dollar (USD), common to Italy andGermany (source: Eurostat);

\footnotetext{
${ }^{1}$ The role of stock market in predicting recession has been largely debated. There are many researches that point out the relevance of stock market in a recession (e.g. see Farmer, 2012).
} 
12.Exchange rate between Euro and Japanese Yen (JPY), common toItaly and Germany (source: Eurostat);

13.Exchange rate between Euro and Sterling (GBP), common to Italy andGermany (source: Eurostat);

14.Exchange rate between Euro and Canadian dollar (CAS), common to Italyand Germany (source: Eurostat);

15.the stock exchange market index (Index), FTSEMIB and DAX, respectively for Italy and Germany (source:

Yahoo Finance market data);

16.the Euro Stoxx 50 index providing a Blue-chip representation in the Eurozone (Stoxx), common to Italy and Germany (source: Yahoo Finance market data).

Table 1: Financial factor variables: $p$-value of ADF test with $p=\sqrt[3]{T-1} \approx 5$ and alternative represented by a model with a linear time trend. For the variables common to both Italy and Germany, a unique value is reported.

\begin{tabular}{lll}
\hline Time-series & Italy & Germany \\
\hline EURIBOR & 0.6648 & \\
B12m & 0.4492 & 0.8319 \\
B10y & 0.4284 & 0.4084 \\
AAA & 0.3609 & \\
BAA & 0.5291 & \\
SprB12mE & 0.3344 & 0.3798 \\
SpreB10yE & 0.7704 & 0.3983 \\
SprAAAE & 0.6578 & \\
SprBAAE & 0.7140 & \\
ER & 0.7560 & \\
USD & 0.6665 & \\
JPY & 0.7168 & \\
GBP & 0.6923 & \\
CAD & 0.3742 & \\
Index & 0.8342 & 0.5354 \\
Stoxx & 0.6340 & \\
\hline
\end{tabular}

The $p$-values of the Augmented Dickey-Fuller (ADF) tests for the 16 variables observed monthly from January 2001 to December 2013 are presented in Table 1. We consider the most complete ADF test formulation, that is $H_{0}: \rho=1$ vs $H_{1}: \rho<1$ for the model including a linear time trend,

$$
\Delta y_{t}=\kappa+(\rho-1) y_{t-1}+\sum_{j=1}^{p} \beta_{j} \Delta y_{t-j}+\delta t+\varepsilon_{t}
$$

All the $p$-values are largely greater than the conventional level (0.05) indicating that all the time-series are nonstationary.

The application of the Dynamic Factor model leads to the estimated loading factors displayed in Table 2 and, as a result, to the estimated factors plotted in Figure 2.1. The factors are both overall decreasing with a peak in 2008. It is interesting to note that the time trend of the German financial factor is very similar to Italy. This is confirmed by the correlation coefficient of the two financial factors equal to 0.996 surely due to the presence of many common variables.

\subsection{The realactivityfactor}

The interaction between real activity and recession is also widely studied in the literature. In order to extract the latent factor we have considered 5 variables:

1. the deseasonalized industrial production index (IPI, source: Eurostat);

2. the harmonized index of consumer prices (HCPI, source: Eurostat); 
Table 2: Financial factor variables: Factor loadings

\begin{tabular}{lll}
\hline Time-series & Italy & Germany \\
\hline EURIBOR & 0.1519 & 0.1529 \\
B12m & 0.1081 & 0.1250 \\
B10y & -0.0272 & 0.1340 \\
AAA & 0.1149 & 0.1122 \\
BAA & 0.0995 & 0.0987 \\
SprB12mE & -0.0989 & 0.0561 \\
SpreB10yE & -0.1578 & -0.0726 \\
SprAAAE & -0.1040 & -0.1079 \\
SprBAAE & -0.1050 & -0.1070 \\
ER & 0.0220 & 0.0296 \\
USD & -0.0197 & -0.0139 \\
JPY & 0.1021 & 0.1075 \\
GBP & 0.1258 & 0.1290 \\
CAD & 0.0500 & 0.0575 \\
Index & 0.1444 & -0.0272 \\
Stoxx & 0.1250 & 0.1196
\end{tabular}
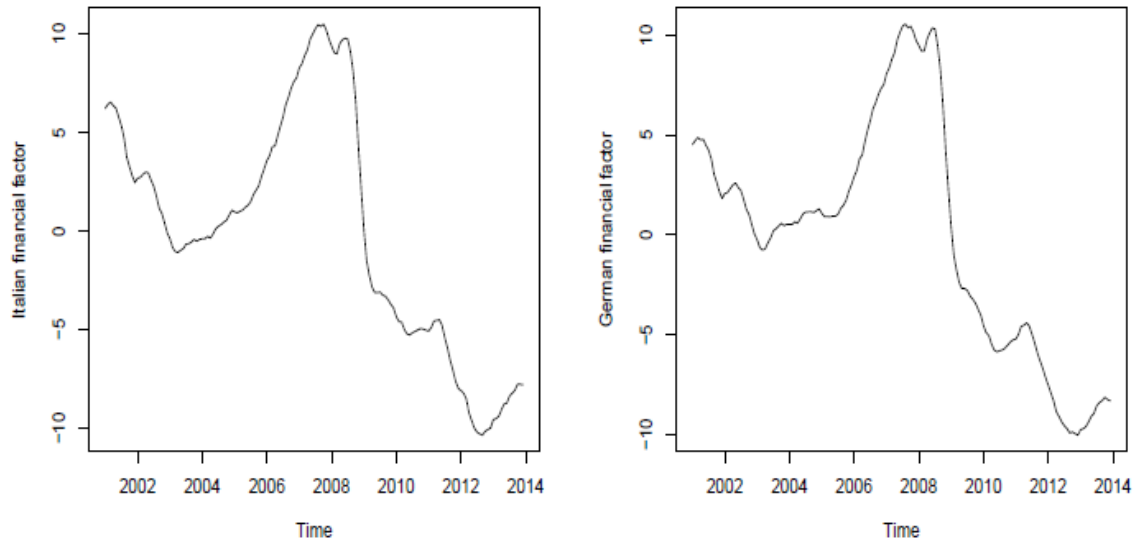

Figure 1: Estimated financial factor for Italy (left) and Germany (right)

3. the deseasonalized manufacturing industrial production index (MIPI, source: Eurostat);

4. the retail trade index (RTI, source: Eurostat);

5. the unemployment rate (UR, source: Eurostat).

All the time-series are clearly non stationary (see Table 3). The loadings are reported in Table 4.

The Italian third factor is almost stable until 2006, then, after a brief rise, it starts to decline since 2008 to the end of the sample period with a slight recovery in 2010.

Table 3: Real activity factor variables: $p$-value of ADF test with $p=5$ and alternative represented by a model with a linear time trend

\begin{tabular}{lll}
\hline Time-series & Italy & Germany \\
\hline IPI & 0.8498 & 0.6959 \\
HCPI & 0.2859 & 0.3749 \\
MIPI & 0.8052 & 0.6798 \\
RTI & 0.6743 & 0.3080 \\
UR & 0.9961 & 0.2602 \\
\hline
\end{tabular}


Table 4: Real activity factor loadings

\begin{tabular}{lll}
\hline Time-series & Italy & Germany \\
\hline IPI & 0.1192 & 0.1708 \\
HCPI & -0.0042 & 0.1090 \\
MIPI & 0.1181 & 0.1726 \\
RTI & 0.1178 & 0.0320 \\
UR & -0.1021 & -0.1242 \\
\hline
\end{tabular}

The behavior of the German real activity factor presents two high peaks in 2008 and 2012. The correlation between the Italian and the German factors is negative $(-0.443)$.

The correlation between the two Italian factors is very high, 0.889 . This feature, in part, suggests that it could be useless to use both the factors altogether, on the other hand, it remarks that the different factors describe the recession in an unambiguous way. On the other hand, the correlation between the two German factors is negative, -0.172 .
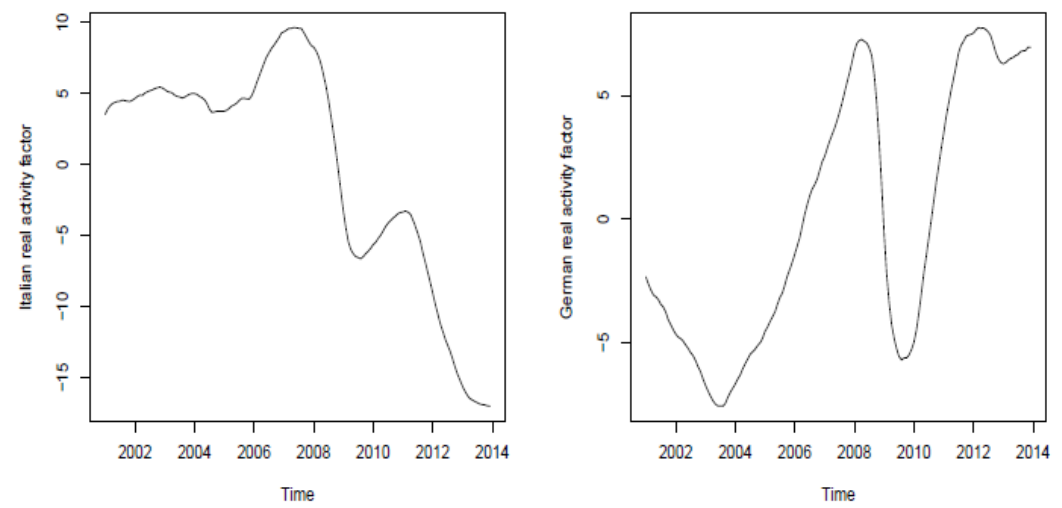

Figure 2: Estimated real activity factor for Italy (left) and Germany (right)

\section{The Kauppi and Saikkonen models}

Kauppi and Saikkonen (2008) have developed some probit models for binary variables. Defined $Y_{t} \in\{0,1\}$ the binary time series at time $t$, the common framework is the assumption that the expected value at the time $t$, conditionally on the information at the time $t-1$, is given by

where $\phi(\cdot)$ is the $c d f$ of a standard Normal distribution.

$$
E_{t-1}\left(Y_{t}\right)=\phi\left(\pi_{t}\right)
$$

According to the specification of the equation of $\pi_{t}$, Kauppi and Saikkonen define four different models:

1. The static model considers only some exogenous variables as regressors,

$$
\pi_{t}=\omega+x_{t-k}^{\prime} \beta
$$

where $x_{t-k}$ is the $p \times 1$ vector of regressors, $k$ is an integer such that $k \geq 0$ and $\beta$ is a $p \times 1$ vector of parameters. Given the observed time series $y_{t}$ for $t=1, \ldots, T$, the generic $h$-step-ahead prediction is easy to compute,

$$
E_{T}\left(Y_{T+h}\right)=\phi\left(\omega+x_{T+h-k}^{\prime} \beta\right)
$$

In particular when $h \leq k$, the exogenous variables are known.

2. The dynamic model includes the one-lagged binary variable,

The one-step-ahead prediction is simply given by

$$
\pi_{t}=\omega+x_{t-k}^{\prime} \beta+\delta y_{t-1}
$$

while for $h>1$ we need $E_{T}\left(Y_{T+h-1}\right)$.

$$
E_{T}\left(Y_{T+1}\right)=\phi\left(\omega+x_{T+1-k}^{\prime} \beta+\delta y_{T}\right)
$$

For a generic $h$,

$$
\begin{aligned}
E_{T}\left(Y_{T+h}\right)= & E_{T}\left(\phi\left(\omega+x_{T+h-k}^{\prime} \beta+\delta Y_{T+h-1}\right)\right)= \\
& =\phi\left(\omega+x_{T+h-k}^{\prime} \beta+\delta\right) P_{T}\left(Y_{T+h-1}\right)+\phi\left(\omega+x_{T+h-k}^{\prime} \beta\right)\left(1-P_{T}\left(Y_{T+h-1}\right)\right)
\end{aligned}
$$

3. The autoregressive probit model encounters the one-lagged value of $\pi_{t}$, 


$$
\pi_{t}=\omega+x_{t-k}^{\prime} \beta+\alpha \pi_{t-1}
$$

The one-step-ahead prediction is given by

and for a generic $h$,

$$
E_{T}\left(Y_{T+1}\right)=\phi\left(\omega+x_{T+1-k}^{\prime} \beta+\alpha \pi_{T}\right)
$$

$$
\begin{aligned}
E_{T}\left(Y_{T+h}\right)=E_{T} & \left(\phi\left(\omega+x_{T+h-k}^{\prime} \beta+\delta \pi_{T+h-1}\right)\right)= \\
\phi & \left(\omega+x_{T+h-k}^{\prime} \beta+\sum_{j=0}^{h-1} \alpha^{j}\left(\omega+x_{T-j}^{\prime} \beta\right)+\alpha^{h} \pi_{T}\right)
\end{aligned}
$$

4. The dynamic autoregressive probit model is the complete model whichincludes exogenous variables, the onelagged binary variable and the one-lagged value of $\pi_{t}$,

$$
\pi_{t}=\omega+x_{t-k}^{\prime} \beta+\delta y_{t-1}+\alpha \pi_{t-1}
$$

The predictions can be easily obtained from the previous models.

\section{DynamicFactormodelsestimation}

The Dynamic Factor models are simply probit models with one or more factors as explanatory variables,

$$
E_{t-1}\left(Y_{t}\right)=\phi\left(x_{t}^{\prime} \alpha\right)
$$

where $x_{t}$ is the $(k+1) \times 1$ vector of explanatory variables including at least one factor while the first element is 1 and $\alpha$ is the $(k+1) \times 1$ vector of parameters. The complete lists of the estimated models for Italy and Germany are reported, respectively in Tables 5 and 6 . The estimation is made by maximum likelihood.

Firstly, we estimate the model with one out of two factors and then the complete model, that is the model with both the factors. The models are characterized by the followingexplanatoryvariables:

- 12-months-lagged factor 1 (model 1);

- 12-months-lagged factor 2 (model 2);

- 12-months-lagged factors 1 and 2 (model 3).

Tables 7 and 8 show the estimates of the three models for Italy and Germany, and some statistics to evaluate the in-sample performance. For Italian recessions, model 1 shows a poor performance, while models 2 and 3 are almost equivalent, even if their statistics cannot be considered fully satisfactory. The $R_{M F}^{2}$ 's are slightly over 0.100 and $R_{M L}^{2}$ reaches 0.150 when both the factors are included. In terms of AIC, model 3 appears to be preferable even if it has an additional parameter with respect to model 2. For German recessions, the first and third models are certainly more impressive, in particular model 3 with all the factors reaches the lowest AIC and shows $R_{M F}^{2}=0.569$ and $R_{M L}^{2}=0.427$. The model with the second factor is definitively poor.

In order to improve the in-sample performance of the Dynamic Factor models we introduce some variable supporting the explanation of the recession. To select a supporting variable, we estimate four additional models considering some candidates variables that are usually considered relevant in the analysis of recessions. The four models we have estimated include the following explanatory variables:

- the 12-months-lagged spread between 10-years BTP and Bund (SprBB) for Italy and between Bund and EURIBOR (SprBE) for Germany (model4);

- the 12-months-lagged ECB rate, already named EURIBOR (model 5);

- the 12-months-lagged FTSEMib for Italy and DAX for Germany (model6);

- the 12-months-lagged unemployment rate (model 7).

The estimates (see Tables 9 and 10) favor the variable Spread BTP/Bund for Italy and the variable EURIBOR for Germany. So, we have enriched the models with the factors including the variables Spread BTP/Bund and EURIBOR, respectively, to the Italian and German recession models. Tables 11 and 12 report the estimates of these models which show very good in-sample performances.In particular for Italian recessions the best model is undoubtedly model 10 . This implies that all the factors continue to be relevant even when the model includes the spread BTP/Bund. A competitive model is model 9, while model 8 is distant in terms of performance. 
Also for German recessions, the best model is model 10 that is the model which includes the two factors and the additional variable EURIBOR. Both the

$R_{M F}^{2}$ and the $R_{M L}^{2}$ reach high values $(0.710$ and 0.501$)$.

However, the comparison with the Kauppi and Saikkonenapproach is not completely favorable. In Table 13 the in-sample statistics of the four models are reported, and the Dynamic and the Dynamic Autoregressive models show a better performance with respect to the estimated dynamic factor models for the Italian recessions. German recessions are also better described by the Dynamic and the Dynamic Autoregressive models.

\subsection{Out-of-sample analysis}

An extensive out-of-sample analysis has been carried out considering three different forecasting horizon: 1 month, 6 months and 1 year. The forecasts have been computed carrying out recursive estimates of the models. The first estimate has considered the data from the beginning (January 2002) until December 2007. Then the first month is dropped out while a new month is added. In such a way, for 1-month horizon, the forecasting period starts in January 2008 until December 2014, so we have collected 84 predictions, for 6-months horizon the predictions start in July 2008 (for a total of 78 predictions) and finally the 1-year predictions are 72, starting from December 2008.

The statistics we have computed are the popular Mean Absolute Prediction Error (MAPE) and Root Mean Square Prediction Error (RMSPE). Moreover we have added the Proportion of Correct Forecasts (PCF): a forecast is evaluated correct if the predicted probability of recession is over 0.50 and the actual value of the time series is 1 , or if the predicted probability below 0.50 is associated with a actual value of the time series equal to 0 . Tables 15 and 16 report the mentioned statistics for a subset of models applied to Italian and German recessions, respectively. In particular, we have considered all the models with the factors and the Dynamic and the Dynamic Autoregressive among those proposed by Kauppi and Saikkonen. For the very short 1-month prediction horizon the results clearly favor the Dynamic Autoregressive model for both Italy and Germany. When we consider the 6months and 12-months horizons, according to MAPE e RMSPE the Italian recessions are best predicted by model 10 including the two factors and the spread BTP/Bund. The PCF is higher for model 10 when the forecast horizon is biannual, while model 9 ensures the highest PCF with a yearly forecast horizon. On the other hand, the most accurate predictions of German recessions can be obtained using model 3, including only the two factors. A competitor is Model 10 (the same variables plus the EURIBOR) in terms of PCF. So, the inclusion of EURIBOR variable allows to reach the highest PCF in the two forecast horizons longer than 1 month. Figures 4.1 and 4.1 show the predictions for forecasts horizon larger than 1 month and actual recessions for the best Dynamic Factor model and the Dynamic Autoregressive model.

So, the general result is an improvement of the forecasts in the horizons larger than 1-month using a model based on dynamic factors. We can also note that for the Italian time-series the statistics naturally deteriorate when the forecast horizon increases. Instead, for German recession the worsening is observed only moving from 1-month to 6-months horizon. In the longest prediction horizon, we can observe a clear improvement of the statistics, probably due to the use of 12-months lagged predictors included in the models.
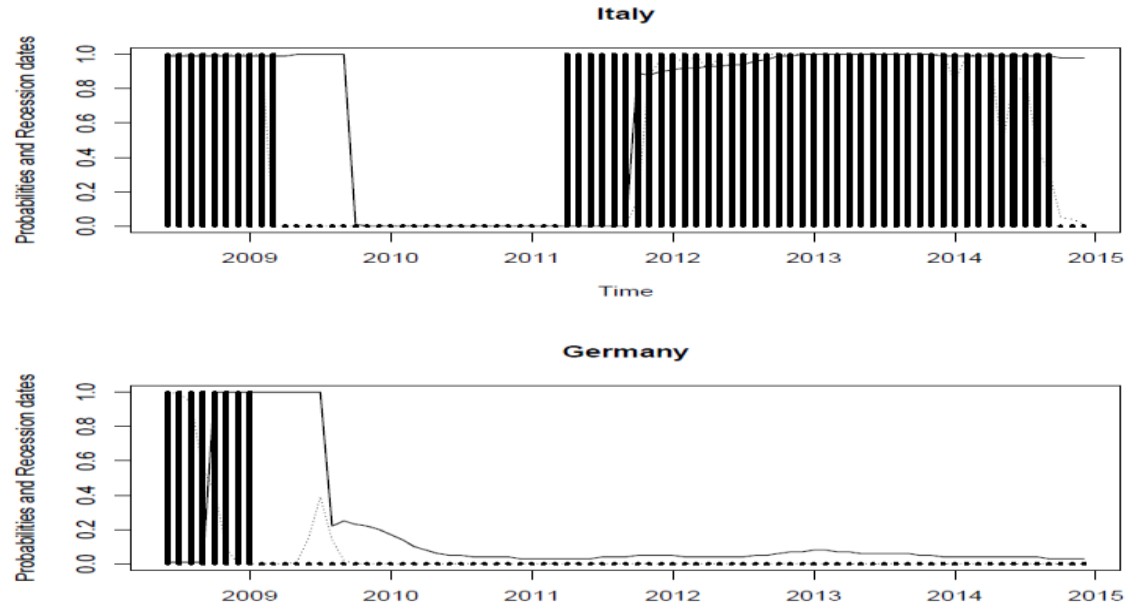

Figure 3: Italy(Dynamic Autoregressive: solid line; Mod3: dotted line) and Germany(Dynamic Autoregressive: solid line; Mod10: dotted line) 6-months ahead prediction. 

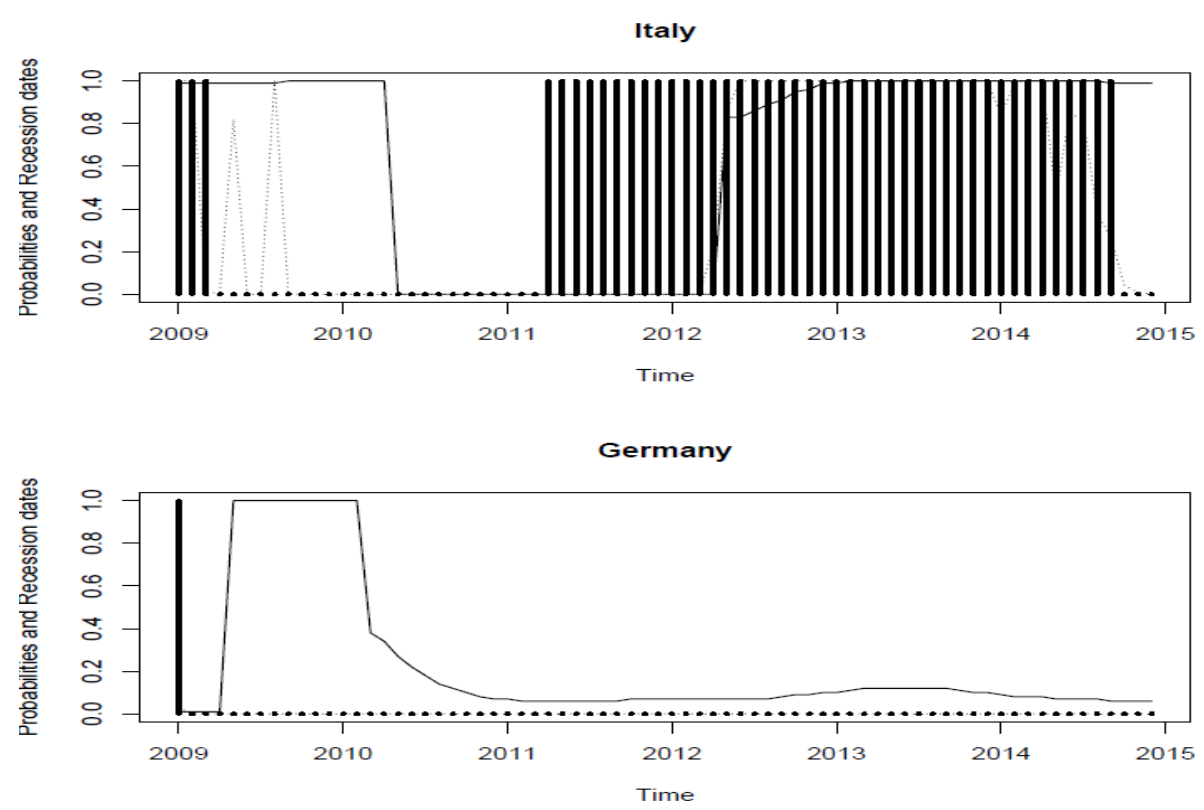

Figure 4: Italy(Dynamic Autoregressive: solid line; Mod3: dotted line) and Germany(Dynamic Autoregressive: solid line; Mod10: dotted line) 12-months ahead prediction.

\section{Concludingremarks}

The prediction of recession periods is a fundamental task of financial and economic authorities supporting the political decisions. A statistical model for fitting and predicting recessions has to take into account the most important economic and financial variables. The high number of possible candidate variables requires a selection process or a transformation into a new limited set of factors. This latter approach is more recent in the literature and is going to provide good results. In this paper a dynamic factor model for Italian and German recessions has been studied after estimating two factors: a financial factor and a real activity factor. The models, possibly enriched with additional explanatory variables, are compared to the models proposed by Kauppi and Saikkonen (2008). While the in-sample fit tends to favor the approach of Kauppi and Saikkonen, in the out-of-sample exercise the better performance of models including dynamic factors is indisputable at forecast horizons larger than 1-month.

\section{Appendix}

Estimation of models and all the data analyses were done using MARSS package (Holmes et al., 2014) implemented in R statistical software. The package is available on the CRAN package repository (www.cran.rproject.org) while codes used to obtain reported results and all additional information useful to make research reproducible will be made available by the authors on request. Data employed are freely available from sources reported in Sections 2.1 and 2.2

Table 5: models for Italian recessions

\begin{tabular}{ll}
\hline Model & Predictors \\
\hline 1 & Factor 1 \\
2 & Factor 2 \\
3 & Factor 1, Factor 2 \\
4 & SprBB \\
5 & ECBrate \\
6 & FTSEMib \\
7 & Unemployment \\
8 & Factor 1, SprBB \\
9 & Factor 2, SprBB \\
10 & Factor 1, Factor 2, SprBB \\
\hline
\end{tabular}


Table 6: models for German recessions

\begin{tabular}{ll}
\hline Model & Predictors \\
\hline 1 & Factor 1 \\
2 & Factor 2 \\
3 & Factor 1, Factor 2 \\
4 & SprBE \\
5 & ECBrate \\
6 & FTSEMib \\
7 & Unemployment \\
8 & Factor 1, ECBrate \\
9 & Factor 2, ECBrate \\
10 & Factor 1, Factor 2, ECBrate \\
\hline
\end{tabular}

Table 7: Italy: in-sample statistics for models 1-3.

\begin{tabular}{llll}
\hline & Mod 1 & Mod 2 & Mod 3 \\
\hline $\mathrm{R}^{2}{ }_{M F}$ & 0.063 & 0.109 & 0.120 \\
$\mathrm{R}^{2}{ }_{M L}$ & 0.082 & 0.137 & 0.150 \\
MAE & 0.438 & 0.410 & 0.406 \\
RMSE & 0.464 & 0.449 & 0.450 \\
AIC & 201.180 & 191.489 & 191.119 \\
LL & -98.59 & -93.745 & -92.56 \\
\hline
\end{tabular}

Table 8: Germany: in-sample statistics for models 1-3.

\begin{tabular}{llll}
\hline & Mod 1 & Mod 2 & Mod 3 \\
\hline $\mathrm{R}_{\text {MF }}$ & 0.251 & 0.010 & 0.569 \\
$\mathrm{R}_{\text {ML }}^{2}$ & 0.218 & 0.010 & 0.427 \\
MAE & 0.239 & 0.308 & 0.135 \\
RMSE & 0.354 & 0.393 & 0.262 \\
AIC & 118.467 & 155.141 & 71.854 \\
LL & -57.223 & -75.570 & -32.927 \\
\hline
\end{tabular}

Table 9: Italy: in-sample statistics for models 4-7.

\begin{tabular}{lllll}
\hline & Mod 4 & Mod 5 & Mod 6 & Mod 7 \\
\hline $\mathrm{R}^{2}{ }_{M F}$ & 0.321 & 0.038 & 0.036 & 0.025 \\
$\mathrm{R}^{2}{ }_{M L}$ & 0.351 & 0.049 & 0.047 & 0.033 \\
MAE & 0.297 & 0.457 & 0.457 & 0.464 \\
RMSE & 0.386 & 0.477 & 0.476 & 0.481 \\
AIC & 147.004 & 206.555 & 206.919 & 209.182 \\
LL & -71.502 & -101.278 & -101.460 & 102.591
\end{tabular}


Table 10: Germany: in-sample statistics for models 4-7.

\begin{tabular}{lllll}
\hline & Mod 4 & Mod 5 & Mod 6 & Mod 7 \\
\hline $\mathrm{R}^{2}{ }_{\mathrm{MF}}$ & 0.035 & 0.380 & 0.012 & 0.000 \\
$\mathrm{R}^{2}{ }_{\mathrm{ML}}$ & 0.034 & 0.311 & 0.011 & 0.000 \\
$\mathrm{MAE}$ & 0.300 & 0.198 & 0.307 & 0.311 \\
$\mathrm{RMSE}$ & 0.389 & 0.324 & 0.392 & 0.394 \\
$\mathrm{AIC}$ & 151.344 & 98.720 & 154.965 & 156.666 \\
$\mathrm{LL}$ & -73.672 & -47.36 & -75.483 & -76.333 \\
\hline
\end{tabular}

Table 11: Italy: in-sample statistics for models 8-10.

\begin{tabular}{llll}
\hline & Mod 8 & Mod 9 & Mod 10 \\
\hline $\mathrm{R}^{2}{ }_{\mathrm{MF}}$ & 0.445 & 0.644 & 0.666 \\
$\mathrm{R}_{\text {ML }}^{2}$ & 0.451 & 0.580 & 0.593 \\
MAE & 0.247 & 0.146 & 0.135 \\
RMSE & 0.360 & 0.260 & 0.247 \\
AIC & 122.896 & 80.974 & 78.373 \\
LL & -58.448 & -37487 & -35.186 \\
\hline
\end{tabular}

Table 12: Germany: in-sample statistics for models 8-10.

\begin{tabular}{llll}
\hline & Mod 8 & Mod 9 & Mod 10 \\
\hline $\mathrm{R}^{2}{ }_{M F}$ & 0.407 & 0.580 & 0.710 \\
$\mathrm{R}_{\text {ML }}$ & 0.329 & 0.433 & 0.501 \\
MAE & 0.186 & 0.132 & 0.089 \\
RMSE & 0.312 & 0.264 & 0.208 \\
AIC & 96.549 & 70.171 & 52.329 \\
LL & -45.274 & -32.085 & -22.164 \\
\hline
\end{tabular}

Table 13: Italy: in-sample statistics for Kauppi and Saikkonnen models.

\begin{tabular}{lllll}
\hline & Static & Dynamic & Autoregressive & $\begin{array}{l}\text { Dynamic } \\
\text { Autoregressive }\end{array}$ \\
\hline $\mathrm{R}^{2}{ }_{\text {MF }}$ & 0.321 & 0.827 & 0.325 & 0.828 \\
$\mathrm{R}^{\mathrm{ML}}$ & 0.351 & 0.672 & 0.355 & 0.673 \\
$\mathrm{MAE}$ & 0.297 & 0.053 & 0.253 & 0.039 \\
$\mathrm{RMSE}$ & 0.386 & 0.386 & 0.397 & 0.163 \\
$\mathrm{AIC}$ & 147.004 & 42.437 & 148.025 & 44.181 \\
$\mathrm{LL}$ & -71.502 & -18.219 & -71.013 & -18.09 \\
\hline
\end{tabular}


Table 14: Germany: in-sample statistics for Kauppi and Saikkonnen models.

\begin{tabular}{lllll}
\hline & Static & Dynamic & Autoregressive & $\begin{array}{l}\text { Dynamic } \\
\text { Autoregressive }\end{array}$ \\
\hline $\mathrm{R}^{2}{ }_{\mathrm{MF}}$ & 0.035 & 0.830 & 0.066 & 0.831 \\
$\mathrm{R}^{2} \mathrm{ML}$ & 0.034 & 0.556 & 0.062 & 0.557 \\
$\mathrm{MAE}$ & 0.300 & 0.040 & 0.624 & 0.033 \\
$\mathrm{RMSE}$ & 0.389 & 0.142 & 0.644 & 0.142 \\
$\mathrm{AIC}$ & 151.344 & 31.904 & 148.7 & 33.776 \\
$\mathrm{LL}$ & -73.672 & -12.952 & -71.35 & -12.888 \\
\hline
\end{tabular}

Table 15: Italy: out-of-sample statistics for some selected models.

\begin{tabular}{|c|c|c|c|c|c|c|c|c|c|}
\hline & & $\begin{array}{l}\text { Mod } \\
1\end{array}$ & $\begin{array}{l}\text { Mod } \\
2\end{array}$ & $\begin{array}{l}\text { Mod } \\
3 \\
\end{array}$ & $\begin{array}{l}\text { Mod } \\
8\end{array}$ & $\begin{array}{l}\text { Mod } \\
9\end{array}$ & $\begin{array}{l}\text { Mod } \\
10\end{array}$ & Dynamic & DynamicAutoreg. \\
\hline \multirow{3}{*}{$\mathrm{h}=1$} & MAE & 0.428 & 0.342 & 0.33 & 0.194 & 0.125 & 0.102 & 0.039 & 0.039 \\
\hline & RMSE & 0.569 & 0.503 & 0.506 & 0.393 & 0.297 & 0.273 & 0.189 & 0.189 \\
\hline & PCF & 0.548 & 0.643 & 0.667 & 0.798 & 0.905 & 0.929 & 0.964 & 0.964 \\
\hline \multirow{3}{*}{$h=2$} & MAE & 0.507 & 0.411 & 0.458 & 0.212 & 0.19 & 0.133 & 0.2 & 0.204 \\
\hline & RMSE & 0.648 & 0.573 & 0.624 & 0.435 & 0.399 & 0.335 & 0.436 & 0.435 \\
\hline & $\mathrm{PCF}$ & 0.443 & 0.57 & 0.532 & 0.772 & 0.848 & 0.861 & 0.81 & 0.81 \\
\hline \multirow{3}{*}{$h=3$} & MAE & 0.597 & 0.507 & 0.554 & 0.266 & 0.26 & 0.249 & 0.381 & 0.381 \\
\hline & RMSE & 0.725 & 0.659 & 0.701 & 0.502 & 0.486 & 0.477 & 0.609 & 0.608 \\
\hline & PCF & 0.315 & 0.466 & 0.438 & 0.74 & 0.767 & 0.74 & 0.63 & 0.63 \\
\hline
\end{tabular}

Table 16: Germany: out-of-sample statistics for some selected models.

\begin{tabular}{llllllllll}
\hline & & Mod & Mod & Mod & Mod & Mod & Mod & \multirow{2}{*}{ Dynamic } & DynamicAutoreg \\
\hline \multirow{4}{*}{$\mathrm{h}=1$} & MAE & 0.155 & 0.312 & 0.087 & 0.109 & 0.215 & 0.092 & 0.266 & 0.035 \\
& RMSE & 0.275 & 0.346 & 0.238 & 0.241 & 0.355 & 0.238 & 0.321 & 0.155 \\
& PCF & 0.869 & 0.869 & 0.929 & 0.893 & 0.762 & 0.905 & 0.881 & 0.976 \\
& MAE & 0.148 & 0.358 & 0.059 & 0.099 & 0.248 & 0.069 & 0.278 & 0.179 \\
$\mathrm{~h}=2$ & RMSE & 0.277 & 0.4 & 0.212 & 0.235 & 0.418 & 0.228 & 0.368 & 0.363 \\
& PCF & 0.785 & 0.785 & 0.899 & 0.911 & 0.772 & 0.937 & 0.873 & 0.873 \\
& MAE & 0.127 & 0.331 & 0.027 & 0.082 & 0.166 & 0.027 & 0.304 & 0.244 \\
$\mathrm{~h}=3$ & RMSE & 0.248 & 0.352 & 0.163 & 0.208 & 0.319 & 0.164 & 0.427 & 0.418 \\
& PCF & 0.877 & 0.89 & 0.973 & 0.904 & 0.89 & 0.973 & 0.822 & 0.836 \\
\hline
\end{tabular}

\section{References}

Chauvet M. and Potter S. (2005), Forecasting recessions using the yield curve, Journal of Forecasting, Volume 24, Issue 2, 77-103.

Chen Z., Iqbal A., and Lai H. (2011), Forecasting the probability of US recessions: a Probit and Dynamic Factor modelling Approach, Canadian Journal of Economics, Volume 44, Issue 2, 651-672.

Estrella A. and Mishkin F.S. (1998) Predicting U.S. Recessions: Financial Variables As Leading Indicators. Review of Economics and Statistics, Volume 80, Issue 1, 45-61. 
Farmer R.E.A. (2012), The Stock Market Crash of 2008 Caused the Great Recession: Theory and Evidence, Journal of Economic and Dynamics Control, Volume 35, Issue 5, 693-707.

Fornaro P. (2016), Forecasting U.S. Recessions with a Large Set of Predictors. Journal of Forecasting, Volume 35, Issue, 6, 477-492.

Fossati S. (2015), Forecasting U.S. Recessions with Macro Factors. AppliedEconomics. Volume 47, Issue 53, $5726-5738$.

Holmes E. E., Ward E. J. and Scheuerell M. D. (2014) Analysis of multivariate time series using the MARSS package.Version 3.9. https://cran.rproject.org/web/packages/MARSS/vignettes/UserGuide.pdf

Kauppi H. and Saikkonen P. (2008) Predicting U.S. Recessions with Dynamic Binary Response models. Review of Economics and Statistics, Volume 90, Issue 4, 777-791.

Nyberg H. (2010) Dynamic Probit models and Financial Variables in Recession Forecasting. Journal of Forecasting, Volume 29, Issue 1-2, 215-230.

Startz R. (2008) Binomial Autoregressive Moving Average models With an Application to U.S. Recessions. Journal of Business and Economic Statistics, Volume 26, 1-8. 